\title{
SPATIAL ELECTRIC LOAD FORECASTING USING AN EVOLUTIONARY HEURISTIC
}

\author{
E. M. Carreno*
}

A. Padilha-Feltrin*

\author{
A. G. Leal ${ }^{\dagger}$ \\ ${ }^{*}$ Faculdade de Engenharia de Ilha Solteira, UNESP - Univ Estadual Paulista, \\ Departamento de Engenharia Elétrica CP 31 \\ CEP 15385-000 - Ilha Solteira SP \\ ${ }^{\dagger}$ ELUCID SOLUTIONS S/A \\ Av Angélica, 2318 Mezanino \\ CEP 01228-904 São Paulo SP
}

\begin{abstract}
A method for spatial electric load forecasting using elements from evolutionary algorithms is presented. The method uses concepts from knowledge extraction algorithms and linguistic rules' representation to characterize the preferences for land use into a spatial database. The future land use preferences in undeveloped zones in the electrical utility service area are determined using an evolutionary heuristic, which considers a stochastic behavior by crossing over similar rules. The method considers development of new zones and also redevelopment of existing ones. The results are presented in future preference maps. The tests in a real system from a midsized city show a high rate of success when results are compared with information gathered from the utility planning department. The most important features of this method are the need for few data and the simplicity of the algorithm, allowing for future scalability.
\end{abstract}

KEYWORDS: Spatial electric load forecasting, land use, knowledge extraction, distribution planning.

Artigo submetido em 10/07/2009 (Id.: 01014)

Revisado em 18/11/2009, 10/04/2010

Aceito sob recomendação do Editor Associado Prof. Ivan Nunes Da Silva

\section{INTRODUCTION}

During the last few decades, the growth of emerging economies, such as some countries in South America and Asia, has enabled their populations to improve living standards; this phenomenon means that every year more inhabitants of these countries look for new homes. This, in tandem with increasing migration from countryside to cities (especially in Asian cities), has created a high level of city expansion. Distribution utilities are currently facing new challenges when planning a network expansion that offers a reliable and economical service inside their service zone.

This situation is more difficult for decentralized electric market structures, where distribution utilities should now deal with profitable operations while considering aging electrical structures owing to the lack of investments during the centralized era.

Over the last few years, research in the distribution area has increased substantially, and attempts to address these growing problems. In many countries, the distribution sector is going through a deep restructuring owing in part to the search for improved quality service levels demanded by consumers in new market-driven structures (Rudnick et alii, 2007). Because of this, over the last few years, research in the distribu- 
tion area has increased substantially to address these growing problems.

To improve the distribution network expansion and planning by considering these factors, distribution utilities should consider spatial electric load forecasting as business priority. In the particular case of Brazil, a new regulatory law for Electrical Distribution Utilities, called PRODIST, imposes the use of Spatial Electric Load Forecasting in their studies (ANEEL, 2008).

Spatial electric load forecasting is a process that tries to determine the future load growth for an electric energy distribution utility (Lee, 2002). This process, apart from answering the question of how much load growth is expected in a determined time frame, also identifies the locations that are most likely to receive the new loads.

Electric load growth inside the service area of an electric utility can be expected for two reasons: first, natural growth because of the natural behavior of existing consumers; and second, new loads because of new consumers. The natural behavior of existing consumers is stationary, with low expected growth; thus, the main reason for load growth is the new consumers inside and outside the actual service zone.

Therefore, the location of these new consumers is of vital importance for the utilities so that they can adopt all the necessary resources for meeting the needs of the future system. Such information can be used to reinforce substations, electric structures, system reconfiguration, and allocation of new substations, among several other activities.

Most of these new consumers will be located outside the service area, continuing the natural growth of the cities, but others will be located inside the service area in zones that are redeveloped. The redevelopment process considers changing the use of some part of the land, generally to accommodate more loads. For example, a zone with horizontal housing could be transformed into a zone with vertical housing, or a residential area could be transformed into a commercial area (Mo-Yuen, Hahn, 1996).

Strategic redevelopment is carried out because of major urban projects looking to improve certain areas of the cities; generally, this kind of redevelopment is planned politically, making it very difficult to forecast. One of the options is using scenario simulations.

Another kind of redevelopment is the growth of the urban core. The urban core acts as an activity center for the entire city, concentrating a large quantity of commercial and residential loads into a relatively small subzone, with financial and cultural offers that attract the rest of the city inhabitants for business or leisure. Because of the concentration of loads, this zone is also the one with higher energy consumption. The growth of the city demands growth of the urban core and its surroundings. The urban core can grow both horizontally and vertically.

This paper deals with a new method for spatial electric load forecasting, by considering new developed zones and redevelopment of the existing ones, using concepts of knowledge extraction algorithms, and elements from evolutionary algorithms, with special emphasis on developing a simple but powerful method with a reasonable use of available data, and taking into account the stochastic nature of new consumers.

\section{OVERVIEW ON SPATIAL LOAD FORE- CASTING}

In specialized literature, spatial electric load forecasting discussion can be traced back to Van Wormer (1954), where the need for a more detailed load forecasting considering the shape of the cities was evidenced. Several works were developed inside electrical utilities, and different techniques were used considering different factors. Most of these works are compiled in (Lee, 2002), which is one of the fundamental sources of information in this area.

The first techniques used pattern recognition to determine preference maps (Lee, Anstos, 1979), where different factors are weighted to determine a specific land use type. This process was later improved using elements from fuzzy logic (Miranda, Monteiro, 2000). Different approaches in fuzzy logic have been developed for this problem, like fuzzy multiobjective decision-making (Chow et alii, 1998), cloud theory (Yang et alii, 2006), ] and recently, statistical approaches (Vazquez et alii, 2008).

Later years have not shown special advances in spatial electrical load forecasting; hence, it is necessary to take advantage of new advances in algorithms to develop new methodologies especially suited to the reality of new cities in emerging countries, considering the requirements of new users and comply with new regulations in the sector.

The spatial electric load forecasting process begins with the data recollection from different sources; these data go from different maps of the utility service area with different information such as land use classification; geographical landscape; socioeconomic distribution; electric structure inventory; historical peak load in different strategic points, like substations and feeders. This information is complemented with that from the different entities entrusted by city planning and information from the utility planning department.

One of the major problems for distribution utilities to carry out a spatial electric load forecasting is the lack of available 
data; hence, the method presented in this work considers that the utilities have access to basic information, like the commercial consumer database and an inventory of all the electrical structures in the system. All these data are organized in a multidimensional database that allows extracting information related to specific zones according to the needs of the chosen method.

After identifying the load growth using conventional methods (Alfares, Nazeeruddin, 2002), the new load is allocated into the service area using the geographical and socioeconomic information from the spatial database. This is one of the most difficult steps in the process because of the stochastic nature of the urban development.

This paper presents an extension of an earlier work (Carreno, Padilha, 2008) by considering new developed zones and redevelopment of the existing ones. The use of concepts of knowledge extraction algorithms and elements from evolutionary algorithms is proposed.

A large database of classification rules obtained automatically by extracting the knowledge available in the spatial database of the city is used to identify user preferences for land use considering elements such as distance to nearby facilities, major roads, and electrical load density.

The redevelopment of the urban core is modeled by considering spatial information from the city's growth as well as user preferences about land use. This proposal models the urban core growth using the database of classification rules to analyze the preferences and dynamics of the urban core related with urban growth, and this information is used to create a preference map for urban core growth. The data, in conjunction with the residential and commercial preference maps, would generate a forecast to determine how the urban core will evolve.

The crossreference of the map's information is made through a stochastic evolutionary heuristic that creates new classification rules using the patterns already recognized in the spatial database.

The method is presented and tested with data from a real midsized city. The results present the preference and forecast maps of the study zone. The validation process of the results is made by comparing the results with the ones obtained in the utility planning department using the known procedure of manual simulation from Lee (2002).

\section{SPATIAL DATABASE}

The availability of data is the most important factor in any methodologies dealing with land use determination in spatial electric load forecasting.
The commercial consumer database available at most distribution utilities identifies all the consumers by name, address, class, consumer code, and historical energy consumption. This information can be crossreferenced with the technical databases using the consumer code, which should be the same in both databases. With both databases, it is possible to allocate energy consumption spatially in the service area.

In case the utility does not have historical records about peak load in substations, a method using statistical daily load profiles can be used to estimate this value from the energy readings in the commercial database (Jardini et alii, 2000).

For the pilot application of this method, a database was built using information including the geographical distribution of all the electric structures inside the service area; utility commercial database of all the consumers with energy consumption from the past years; statistically obtained load curves for all types of consumers in different consumption levels; and a land use classification map of the zone.

This information was crossreferenced, and a multidimensional spatial database was created. This database allows visualizing specific data such as load growth for specific areas, substations, feeders, transformers, utilization factors, installed kVA, spatial load density, and other valuable information.

The spatial resolution adopted was $0.5 \mathrm{~km}^{2}$ to take advantage of the information available, but smaller resolutions are available on demand. Figure 1 presents the map of the service area with a total extension of $180 \mathrm{~km}^{2}$. Each circle represents one subarea of $0.5 \mathrm{~km}^{2}$. Black circles represent currently occupied areas and white circles represent unoccupied areas in zones with the possibility of being occupied. The zones without circles represent the areas without the possibility of development for different reasons.

From the commercial database, it is possible to identify the location of the different structures of interest such as schools, hospitals, and commercial zones, among others. The major roads and zones without potential can be identified from aerial maps, and other information is obtained from the utility planning department, like activity centers, and special urban interest zones, to name a few.

The information used in this work is readily available in most of the electric utilities around the world, allowing fast implementation.

\section{CLASSIFICATION RULES}

To allocate the future loads inside the service area, it is necessary to consider several factors; for example, a pollutant 


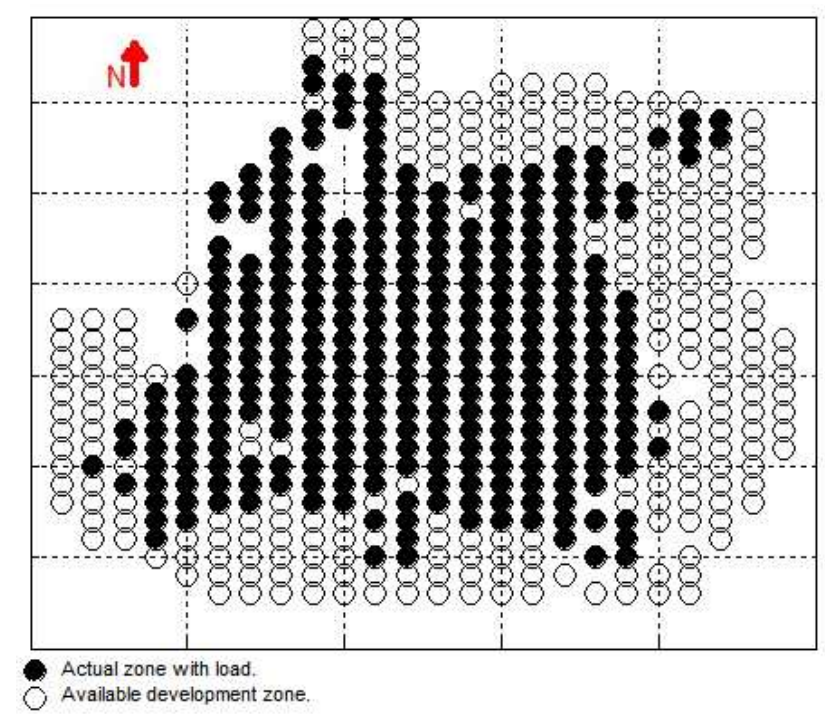

Figure 1: Service area map, aerial view.

industry cannot be allocated near residential zones; residential consumers like to live near other residential zones, but not too far away from commercial zones and not too distant from their workplaces and so on. These preferences are sensitive to location and culture; hence, they are specific for each city. If static rules were adopted, it will be necessary to change them for each study. Some of these new consumers will be allocated in already populated zones having further growth opportunities, while others will be allocated in new zones that will be populated in the midterm with new infrastructure.

The local authorities have made a series of studies related with the city growth; unfortunately, this information is not reliable because of its political dependence. Hence, future growth studies should use this information only as an indicator, not as a rule.

One of the tools used to decide which new zones will be populated in the mid- and long-term in the service area was the classification rules, discussed first in (Chow et alii, 1998), and broadly used in different algorithms since then. In (Miranda, Monteiro, 2000), classification rules were introduced to this problem to determine a land use score; these rules can be expressed as:

(IF Object (1) IS Attribute) AND (IF Object (2) IS Attribute) AND .... THEN (LandScore IS Attribute)

Here, Object refers to a set of elements of interest to be considered in the process, like structures (schools, roads, highways, activity center), nearby zones, or other elements of interest.
Attribute refers to the measure of the relations between the different zones and the elements. If one element is distance to a road, the rules are expressed by near, close, away, faraway. For an element of residential electrical density, the rules are expressed in low, normal, and high. Each Attribute corresponds to a finite and exclusive set in the domain of the corresponding object. For example: All the distances from 0 $\mathrm{km}$ to $1 \mathrm{~km}$ inclusive to a school is considered near; All the distances between $1.01 \mathrm{~km}$ to $5 \mathrm{~km}$ inclusive are considered as medium; and so on. The range of values for each attribute are selected based on an statistical analyses of all the data for each object.

For each object, customers have different preferences, and that is why a different set of attributes exist for each one, for example, the attribute near does not have the same meaning for a school or for the activity center of the city.

\section{CODING OF THE CLASSIFICATION RULES}

To extract and use the knowledge from the spatial database, it is necessary to set up an acceptable coding to efficiently store the information gathered during the process.

The classification rules (Input and Output) are coded as a vector, where each position represents an element of interest, and the value stored represents a linguistic expression suiting to the specific object. All the elements are related with the AND operator. Thus, a vector containing linguistic expressions for a set of objects represents a rule. For example, using the values $\{\mathrm{L} \mathrm{N} \mathrm{H}\}$ to represent three different linguistic references as $\operatorname{Low}(L)$, Normal $(N)$, and $\operatorname{High}(H)$ for each of the objects considered in the classification rule, the rule is:

INPUT (IF Distance to object 1 is $L$, AND Distance to object 2 is $N, \ldots$, AND Residential load density is $H$ ) then OUTPUT (Residential load is H, AND Commercial load is $N$, AND Industrial load is $L)$. This rule can be represented as $(L, N, \ldots, H) \Rightarrow(H, N, L)$, which can be written as the vector $[L N \ldots H][H N L]$.

For this particular application, the Input objects were chosen as distance between the subarea and interest points, and load densities; the load densities are calculated in $3 \times 3$ and $5 \times 5$ subareas around the subarea in study, and the output objects are loads for consumer class.

The input and output objects are not fixed and can comprise any combination of objects, so long as a linguistic definition set for them exists. 


\section{KNOWLEDGE EXTRACTION}

To characterize the service territory, it is necessary to find out the classification rules that will allow us to settle the future land use preferences.

These rules and the defining sets could be determined manually by the planner using rules supported by common sense and practice, however, these rules could work well for one specific city based on the expertise and the knowledge of the planner. The differences between cities around the world demonstrate the need to settle a different set of rules for each one. The method in this paper presents a systematic way to extract a set of rules automatically for each city.

Using the information available from the spatial database, it is possible to create a database of known rules that can be later used to settle the preferences for future areas, considering that the growth of the city is a stationary stochastic process. The procedure for creating such database is as follows:

Being $R 1$ the set of rules for the populated areas with known output,

1. In the spatial database, identify all the elements of interest (Input), like activity centers, schools, major highways and roads, parks, among others;

2. Establish the linguistic expression for each element of interest;

3. For each populated subarea i:

a. Calculate the linguistic expressions for each element of interest, considering the relation of the subarea with the elements of interest and their surroundings, form the linguistic rule and store it in $R 1$.

With this algorithm all the knowledge present in the populated area is extracted and processed.

In some knowledge-extracting algorithms, the known rules will be mixed using different evolutionary operators to create new rules (Yi-Chung et alii, 2003). This approach is not used because most of the rules created will be useless; instead, each unpopulated area is analyzed to find out its output, by optimizing resources and minimizing computational time.

To compare how similar two rules are, the Hamming distance is used. The Similarity $(S M)$ (1) is defined as the Hamming distance between two rules, that is, the number of positions where the corresponding values of the rule Aare different than the ones in rule $B$ (Hamming, 1950).

$$
S M=\sum_{i=1 . . \# \text { Objects }} \mid \text { RuleA }(i)-\text { RuleB }(i) \mid
$$

One way to deal with the rules for the unpopulated area will be to build the input part of the linguistic classification rules and settle the output according to the same part of the rule with the highest $S M$ value among all the rules in $R 1$.

The process is described next:

For each unpopulated area $k$ :

1. Build the input part of the classification rule;

2. Calculate the $S M$ value between the rule in analysis and all the elements in $R 1$;

3. Assign to the output part of the classification rule in analysis, the output of the rule in $R 1$ with the highest $S M$.

In this manner, however, the randomness of the behavior for this particular problem is lost. Besides, owing to the nature of the distribution of the unpopulated areas (generally outside the service area), the $S M$ value could be low and not significant enough to assign a logical output. Another problem arises when several rules with the same input part have different outputs; hence, it is not possible to choose one instead of another only with this information.

To overcome these problems, an evolutionary approach is proposed by selecting the output part of the classification rule with more than one source of similar rules. The output part of the classification rule is determined by selecting out a set of similar rules $(R S M)$, sorting it using the SM value for each one as the fitness function, and applying a uniform crossover between two candidates selected by a tournament. In this way, the output parts of the rules are chosen using the characteristic of this genetic operator as controlled randomness and statistical survival of the fittest.

In a tournament selection, two or more solutions are picked at random and a tournament strategy is used to select one after a number of rounds are played. In each round, a number of solutions come together to compete, and only the fittest solution will win and be selected (Chambers, 1999). For this particular application, two rounds are played, each one with two solutions randomly picked using the $S M$ value as the fitness function.

The crossover operator is one of the most important operators in genetic algorithm. This operator is the one in charge of propagating the genetic information of two elements of the 
current population to the next, by combining their genetic code to generate a descendant.

The complete process is detailed in the following algorithm:

For each unpopulated area $k$ :

1. Build the input part of the classification rule;

2. Calculate the $S M$ value between the rule and all the elements in $R 1$;

3. Build a set of similar rules (RSM) using the $S M$ value;

4. Select two candidates to be crossed over using tournament selection;

5. Crossover the output part of the two candidates selected, and assign that value to the output part of the classification rule in analysis.

The size of the RSM set was determined as 10 for a vector of 13 elements (10 inputs, 3 outputs) by experimentation; with values higher than 10 the $S M$ values are too low, and with lower values the diversification is low. At the end of the process, all the unpopulated areas are classified into different preferences for consumer class and expected load density, creating a preference map where it is possible to identify different areas of interest to the planner.

Considering the stochastic nature of this process, the algorithm is repeated several times to identify the interest areas more clearly.

To explain the idea, a brief example is presented next:

The values $\{L N H\}$ represent three different linguistic references as Low, Normal and High for each of the objects considered in the classification rule.

The classification rule for an unpopulated subarea under analysis is given by:

(Distance to major road is $N$, Distance to nearest school is $N$, Distance to activity center is $L$, Residential density in $3 \times 3$ neighborhood is $L$, Commercial density in $3 \times 3$ neighborhood is $N$, Industrial density in $3 \times 3$ neighborhood is $H$ ).

The vector [NNLLNH] is the input part of the classification rule for the unpopulated area $k$.

For this vector, the RSM set size is determined as 6 for experimentation. The vector is compared with all the elements in the set $R 1$, and an RSM set is formed with 6 elements, as shown in Table 1.
Table 1: RSM Set

\begin{tabular}{|c|c|c|c|}
\hline Element & $S M$ & Input & Output \\
\hline 1 & 5 & {$[N L L L N H]$} & {$[L N H]$} \\
\hline 2 & 5 & {$[H N L L N H]$} & {$[L L H]$} \\
\hline 3 & 3 & {$[N L N L N N]$} & {$[L N N]$} \\
\hline 4 & 4 & {$[L N L N N H]$} & {$[N L H]$} \\
\hline 5 & 3 & {$[L N L N N N]$} & {$[L H L]$} \\
\hline 6 & 4 & {$[L N L L L H]$} & {$[L N H]$} \\
\hline
\end{tabular}

Using a tournament selection, vectors (1) and (3) from the RSM Set presented in Table 1 are selected to be crossed over.

The output parts of rules (1) and (3), [LNH] and [LNN], are mixed using a uniform crossover obtaining the vector $[L N H]$.

At the end, the output part for the rule [NNLLNH] should be $[L N H]$.

With the results obtained until this point, it is enough to make an entry-level spatial electric load forecasting process that is able to identify all the new zones with possibility of growth. But to complement the results and lower the spatial error, it is necessary to consider some level of redevelopment inside the service area.

The redevelopment process considered is the urban core growth. The rule database and the same principles of similarities between zones are used in this step; hence, there is no need for new data or different mechanisms than the ones already developed. The process starts by identifying the activity center by searching for the subareas with the highest values of residential and commercial energy consumption. The location of this special subareas is easy to identify because in the spatial database a zone identified as the urban core in the "elements of interest" already exists.

The classification rules of the subareas in the urban core are compared with the ones in a $3 \times 3$ neighborhood zone, and a score is calculated adding those values. This value represents the relationships between the urban core and its immediate neighbors. Next, the classification rules for all the subareas in the service zone are compared with the ones in the urban core and its own $3 \times 3$ neighborhood zone. When presented graphically, the results of this exercise will show how the different subzones share similarities with the urban core, and especially, how the different factors in the city will take the urban core growth in a specific direction.

An outline of the complete method is presented in Fig. 2. This process is done for each planning horizon. The results 


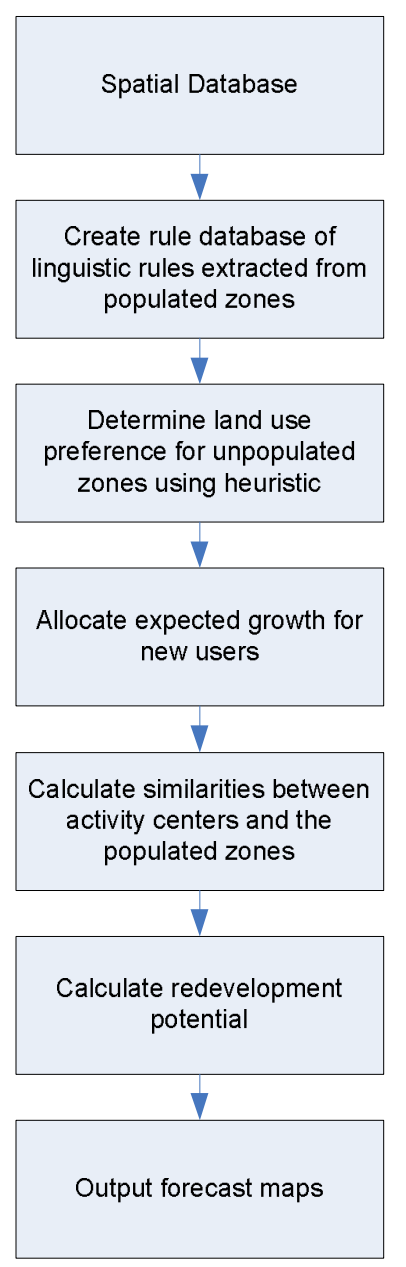

Figure 2: Outline of the complete algorithm

obtained in planning horizon n-1 are used as input elements for planning horizon $\mathrm{n}$.

\section{TESTS AND RESULTS}

The method was incorporated into the UE viewer, a distribution network management software designed for Elucid Solutions, using data from a midsized city in Brazil called Presidente Prudente, in the interior of São Paulo State, with around 200,000 residents and two major activity centers identified as major commercial zones.

Some information obtained about the service area, especially information on future land developments, were not included in the database. This would be used as a validation set for the results.

With the information available, the next objects were chosen to form the classification rules for this particular system:
Input

- Distance to nearest school;

- Distance to nearest hospital;

- Distance to nearest major road;

- Distance to main activity center;

- Distance to secondary activity center;

- Residential load density in a 3x3 neighborhood zone;

- Commercial load density in a 3x3 neighborhood zone;

- Industrial load density in a 3x3 neighborhood zone;

- Residential load density in a 5x5 neighborhood zone;

- Commercial load density in a 5x5 neighborhood zone;

- Industrial load density in a 5x5 neighborhood zone.

Output

- Residential load;

- Commercial load;

- Industrial load.

Figure 3 presents the results obtained for the residential class. In this figure, the circles represent the zones of the service area where some load is already present. The area expected to be occupied according to the information obtained from the utility planning department (validation set) is identified by diamonds, and the area expected to be occupied is identified by squares.

In Fig. 3, one area in the South is identified by the planning department as being occupied for residential consumers in the future but is not identified by the algorithm. This is because a large project is expected to be built in that part of the city, changing the classification of this area from industrial to commercial. Because of the industrial classification of the zone, the algorithm did not consider this area suitable for residential occupation.

In other tests, considering the change of the land use classification and the inclusion of a large simulated load in this zone, it was possible to match this expected behavior.

Another interesting result is the number of areas identified by the algorithm that were not identified by the manual simulation of the utility planning department. This is because the zones forecasted by the planning department were based in 


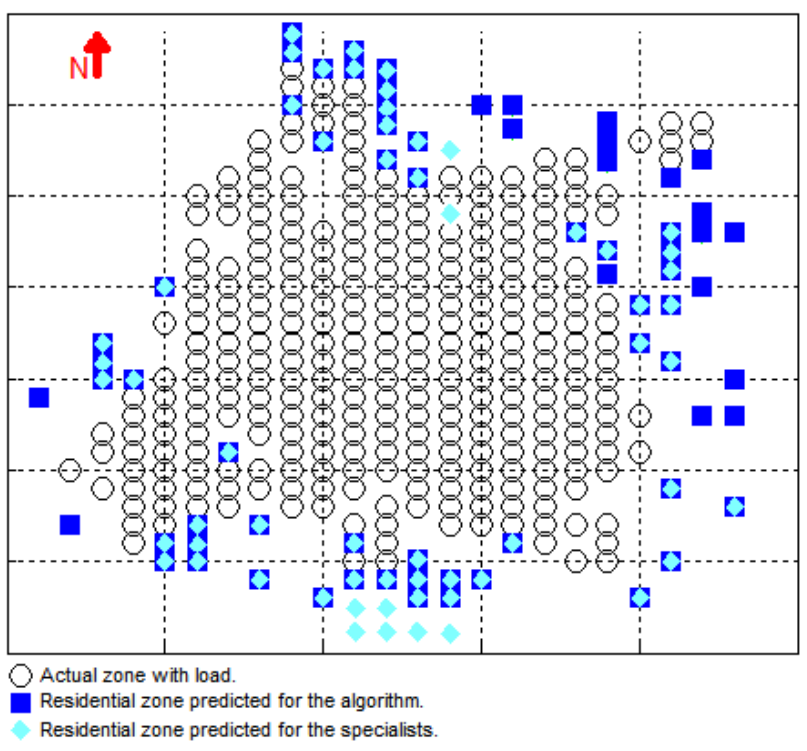

Figure 3: Service area map with expected residential preference.

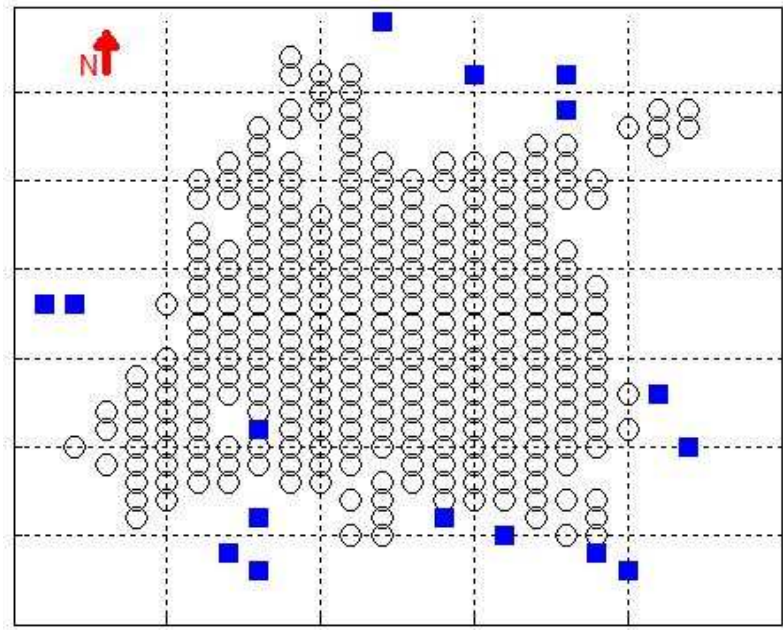

Zone with load.

Expected industrial zone.

Figure 4: Service Area Map with expected industrial preference.

present and future development projects, and not likely the terrain characteristics. Therefore, the answer obtained from the algorithm is more complete because it is possible to identify all the zones of interest in the long term.

The results for the industrial and commercial sector are presented in Figs. 4 and 5, respectively, presenting complementary patterns between the three consumer classes analyzed (see Fig. 6).

Finally, it is necessary to settle the temporal allocation of

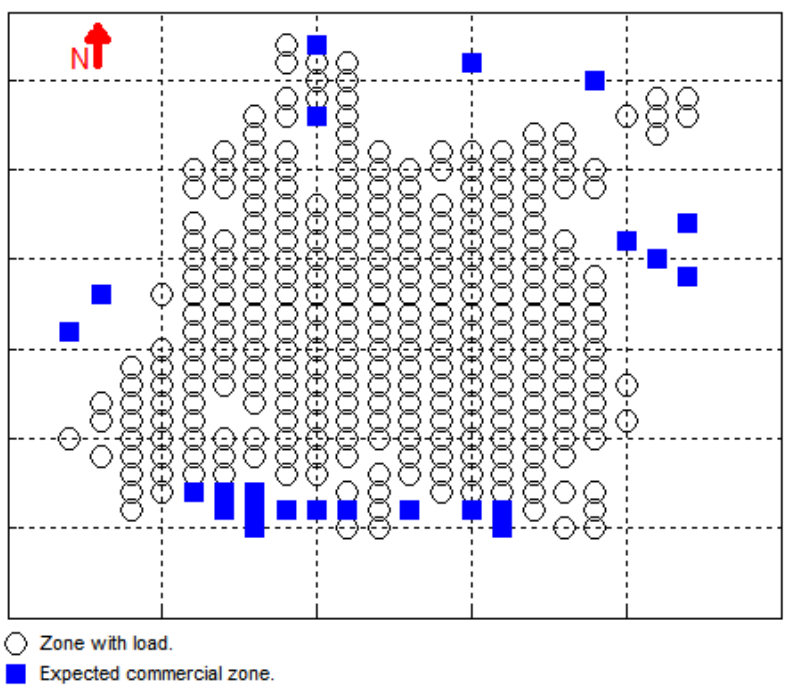

Figure 5: Service Area Map with expected commercial preference.

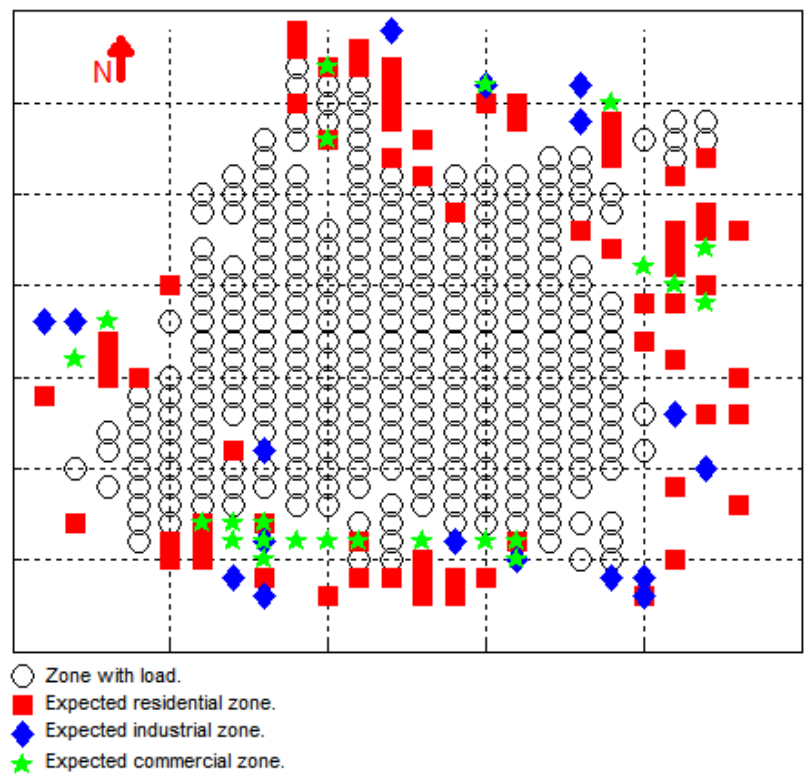

Figure 6: Spatial allocation for the three major classes.

these areas. To do this, after calculating the expected growth because of new consumers in the service area using traditional methods, the areas are assigned considering neighboring characteristics, assigning first the ones with a bigger load density nearby, obtaining the results presented in Fig. 7.

The methodologies used to calculate the load growth are not part of the scope of this work. This load growth was calculated using time series and population data, but any other method could be used to determine this value. 


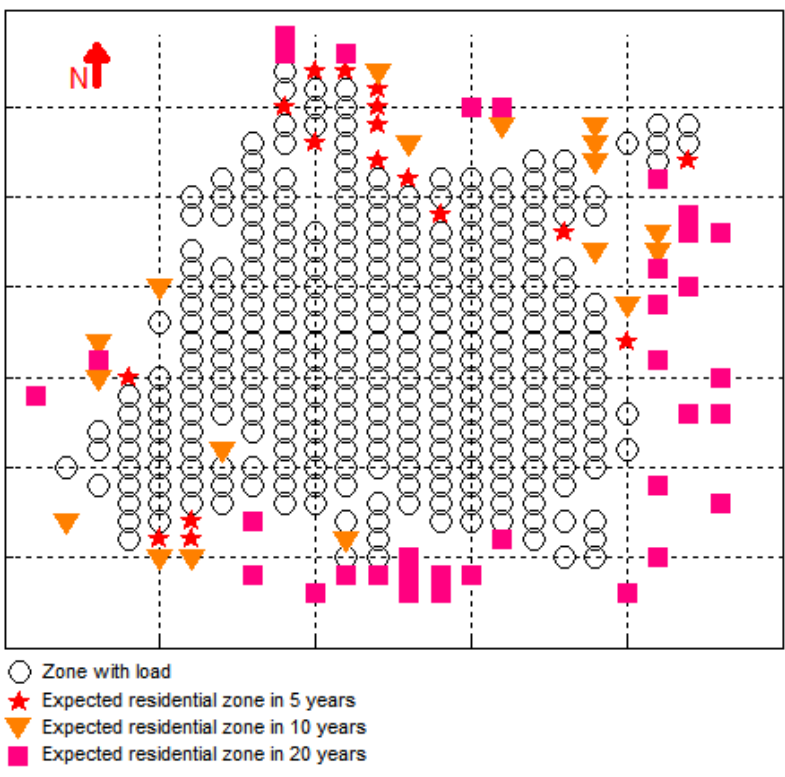

Figure 7: Temporal allocation for residential consumers.

The first areas to be occupied are located in the southeast part of the service area, an area with rapid and constant urban projects for mid- and high economic levels, and in the north of the city, where proximity to another small city and land intended to popularize housing are important factors. This information was not introduced into the algorithm.

Figure 8 shows the redevelopment preference map based on the urban core growth. Obviously, the areas with higher probability of redevelopment are the ones around the urban core, but it is important to note that the urban core is not growing axially around that point, but that it has a strong influence to grow in the southwest. This behavior is explained by the growth in commercial and residential density in that direction, especially for high-income housing. Meanwhile, to the north and east of the urban core, growth is not expected because of the low growth in electrical load density.

To complement the validation of the method, the results were compared with an internal forecast of the electric utility, made manually for the engineers in charge of the network planning, using the manual method presented in (Lee, 2002) , resulting in an error rate between 5\% and 10\% in different simulations the short term ( 5 years) and mid term (10 years). For the long term (20 years) there were no results to compare to. These results are considered very good, specially because the manual simulation from (Lee, 2002) could take easily one month of work for several employees.

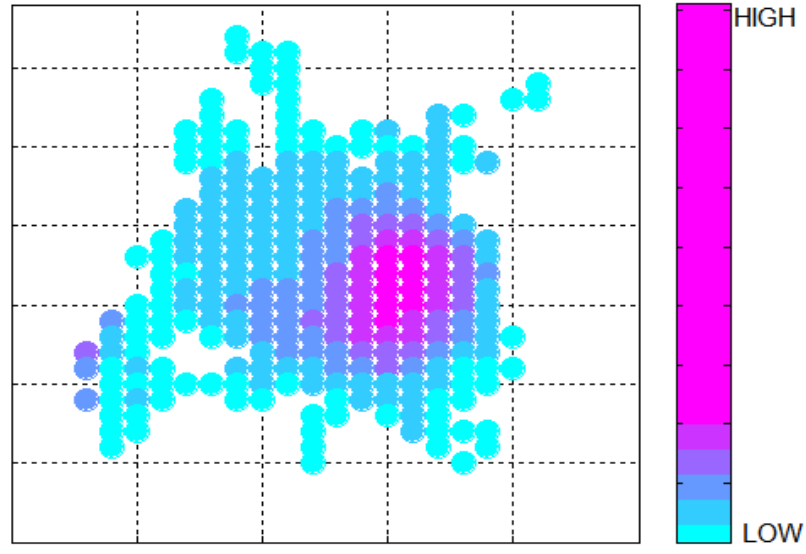

Figure 8: Redevelopment preference map.

\section{CONCLUSIONS}

A simple and efficient algorithm to settle future land use and occupation in the spatial electric load forecasting process has been presented by considering the identification of new zones with potential growth and the redevelopment of the existing ones. This application has been proved to be an important tool in assisting distribution network planning engineers to identify important zones with the possibility of growth inside the service area. The algorithm uses simple procedures to extract the knowledge related to user preferences about land use from a multidimensional spatial database. This knowledge is stored in the form of linguistic classification rules.

It is possible that several classification rules with the same input present different outputs. This is because of the stochastic nature of the process, which makes the use of fixed classification rules a problem. To overcome this difficulty, the consumers' stochastic characteristic is considered using elements from evolutionary algorithms to create new classification rules for the vacant areas using the knowledge previously extracted.

The final result shows the efficiency of the method in creating a forecast map similar in $95 \%$ to the utility planning department forecast, with low computational effort and minimum computer memory requirements, using few resources and data. The results obtained prove the efficacy of the approach and encourage more work in that direction.

One of the main advantages of this method is the reduced set of data needed to execute it, using only the distribution utility commercial database and a georeferenced data set of the network elements. This approach could be helpful to utilities with limited resources and few available data. The multidimensional spatial database created for this application can be used as support information to solve other problems related 
to the planning and operation of the distribution network.

\section{REFERENCES}

Alfares H. K., Nazeeruddin M. "Electric load forecasting: literature survey and classification of methods," International Journal of Systems Science, v.33, no 1, p. 23-24, Jan. 2002.

ANEEL, Procedimentos de Distribuição - PRODIST 2008. Available online http://www. aneel.gov.br/. (In portuguese)

Carreno, E.M.; Padilha-Feltrin, A., "Evolutionary heuristic to determine future land use," in Proc. Power and Energy Society General Meeting, IEEE , pp.1-6, 20-24. Jul. 2008.

Chambers, L. Practical Handbook of Genetic Algorithms: Complex Coding Systems, Volume III. USA, CRC Press, 1999.

Chow, M., Zhu, J., Tram, H., "Application of fuzzy multiobjective decision making in spatial load forecasting," IEEE Trans. on Power Systems, vol 13, pp 1185-1190. Aug. 1998.

Hamming, R. W., "Error detecting and error codes." Bell System Technical Journal, New York, v.26, no 2, p.147160, 1950.

Jardini, J. A., Tahan, C. M., Gouvea, M. R., Ahn, S. U., Figueiredo, F. M., "Daily Load Profiles for Residential, Commercial and Industrial Low Voltage Consumers.," IEEE Trans. on Power Delivery, vol. 15, no. 1, pp 375380, Jan. 2000.

Lee, W, H. Aanstos, J., "Some unique signal processing applications in power systems analysis," IEEE Trans. on Acoustics, Speech, and Signal Processing, v. 27, no 6, pp 685, Dec. 1979.

Lee, W, H. Spatial Electric Load Forecasting, 2nd Edition. New York, Marcel Drekker Inc, 2002.

Miranda, V., Monteiro, C., "Fuzzy inference in spatial load forecasting," in Proc. Power Engineering Society Winter Meeting, pp 1063-1068 vol.2, Jan. 2000.

Mo-Yuen, C., Hahn, T., "Methodology of urban redevelopment considerations in spatial load forecasting," in Proc. Transmission and Distribution Conf, pp 452-457, Sep. 1996.

Rudnick, H.; Arnau, A.; Mocarquer, S.; Voscoboinik, E., "Stimulating efficient distribution," IEEE Power and Energy Magazine, vol.5, no.4, pp.50-67, Jul. 2007.
Van Wormer, F. C., "Some Aspects of Distribution Load Area Geometry," Power Apparatus and Systems, Part III. Transactions of the American Institute of Electrical Engineers, vol.73, no.2, pp.1343-1349, Jan. 1954.

Vasquez-Arnez, R.L.; Jardini, J.A.; Casolari, R.; Magrini, L.C.; Semolini, R.; Pascon, J.R., "A methodology for electrical energy forecast and its spatial allocation over developing boroughs," in Proc Transmission and Distribution Conference and Exposition, 2008. pp.1-6, 2008.

Yang, X., Yuan, J., Zhang, T., Mao, H., "Application of Uncertainty Reasoning Based on Cloud Theory in Spatial Load Forecasting," in Proc. of Intelligent Control and Automation, vol 2, pp 21-23. Jun. 2006.

Yi-Chung, H., Ruey-Shun, C., Gwo-Hshiung, T., "Finding fuzzy classification rules using data mining techniques," Pattern Recognition Letters, no 24, pp 509519, Jan. 2003. 